\title{
Extreme reproductive specialization within ant colonies: some queens produce males whereas others produce workers
}

\author{
ROLF KÜMMERLI \& LAURENT KELLER \\ Department of Ecology and Evolution, University of Lausanne \\ (Received 10 November 2006; initial acceptance 24 February 2007; \\ final acceptance 21 March 2007; published online 1 October 2007; MS. number: 9176)
}

\begin{abstract}
A key feature differentiating animal societies is the partitioning of reproduction among breeders. We studied how reproduction is partitioned among nestmate queens of the ant Formica exsecta in laboratory colonies. In polygynous (multiple-queen) colonies, queens can increase their reproductive success by laying more eggs or by increasing the proportion of eggs that develop into reproductive individuals instead of workers. We found that reproductive skew among queens for egg production was low, but that $84 \%$ of all queens contributed exclusively to one type of brood, either males or workers (no new queens are produced in the laboratory). Furthermore, our data revealed that the degree of reproductive specialization among queens significantly increased during brood development. Contrary to predictions of most reproductive skew models, the extent of reproductive skew was not associated with relatedness among co-breeding queens. We also found no association between the pattern of queen specialization and relatedness between queens and their mates. Such an association would occur if queens mated to related males specialize in male production to avoid the cost of inbreeding. Altogether, our findings show a yet undescribed pattern of reproductive specialization among nestmate queens in ants and emphasizes the need to investigate reproductive contributions of queens for each type of offspring separately.
\end{abstract}

(c) 2007 The Association for the Study of Animal Behaviour. Published by Elsevier Ltd. All rights reserved.

Keywords: ants; breeding system; Formica exsecta; polygyny; reproductive skew; reproductive specialization; social insects

One of the major transitions in evolution is the shift from solitary organisms to societies with reproductive division of labour (Maynard Smith \& Szathmary 1995; Szathmary \& Maynard Smith 1995). A key feature differentiating animal societies is the partitioning of reproduction among individuals (Keller \& Reeve 1994), which can range from strictly equitable breeding (referred to as low-reproductive skew) to extreme skew where reproduction is limited to one or a small subset of breeders.

In social Hymenoptera, reproductive skew is generally very high in colonies containing a single queen because queens typically monopolize most of the reproduction (Bourke 1988; Jeon \& Choe 2003; Hammond \& Keller 2004). In many species, however, colonies may contain two or more fertile queens (Keller 1993b, 1995; Bourke

Correspondence and present address: R. Kümmerli, Institute of Evolutionary Biology, Ashworth Laboratories, West Mains Road, University of Edinburgh, Edinburgh EH9 3JT, Scotland, U.K. (email: rolf. kuemmerli@ed.ac.uk).L. Keller is at the Department of Ecology and Evolution, Biophore, University of Lausanne, CH-1015 Lausanne, Switzerland.
\& Franks 1995), and there is considerable intra- and interspecific variation in the relative contribution of these queens to reproduction (Reeve \& Keller 2001). Most studies conducted to date investigated the extent to which nestmate queens differ in their overall reproductive output (Reeve \& Keller 2001). However, even if queens contribute similar number of eggs, they might differ in their reproductive success (Keller 1993a) because the fitness of nestmate queens does not only depend on their fecundity, but also on the type of egg produced (haploid male or diploid female) and their developmental fate (gyne (new queen) or worker development of diploid eggs).

Several studies revealed that queens in multiple-queen (polygynous) ant colonies may, indeed, differ in their relative contribution to the production of different offspring classes (gynes, males and workers) (Ross 1988; Fournier \& Keller 2001; Rüppell et al. 2002; Fournier et al. 2004; Hammond et al. 2006, Kümmerli \& Keller 2007). Some of these studies also showed trade-offs in the relative contribution of queens to two different classes of offspring (Ross 1988; Fournier \& Keller 2001; Fournier et al. 2004). A significant trade-off between a queen's contribution to 
gyne and worker offspring has been found in Solenopsis invicta (Ross 1988) and Pheidole pallidula (Fournier et al. 2004), while in the Argentine ant Linepithema humile the trade-off is between the production of males and gynes (Fournier \& Keller 2001).

A recent study of the ant Formica exsecta suggested an extreme case of reproductive specialization with an estimate that approximately $79 \%$ of queens appear specialized in the production of either males or workers (Kümmerli \& Keller 2007). However, the high queen number per colony in the population studied did not allow us to determine brood maternity and the estimated degree of queen specialization was based only on colony kin structure.

The first aim of this study was to test under laboratory conditions whether there is high reproductive specialization among nestmate $F$. exsecta queens. In particular, we wanted to test whether some queens show complete specialization in the production of a single offspring class, a pattern that has not yet been firmly documented in any social insect species. The second aim of the study was to identify the proximate mechanism underlying queen specialization. To do so, we compared the degree of reproductive specialization among queens at the egg and pupal stages to test whether reproductive specialization is mediated by some queens producing exclusively or mostly haploid male eggs while other queens produce only or mostly diploid female eggs. This analysis also permitted to test whether queen specialization changed between the egg and the pupal stage with such a change possibly being caused by differential survival of haploid or diploid eggs or by workers manipulating brood composition.

Furthermore, we tested whether the extent of reproductive skew among queens is associated with the relatedness among co-breeders and colony productivity as predicted by reproductive skew models (reviewed in Keller \& Reeve 1994; Johnstone 2000; Reeve \& Keller 2001). There are three different types of reproductive skew models that can be distinguished according to their assumption about who is controlling the allocation of reproduction. The different models assume that the allocation of reproduction is either controlled by the dominant (concession models), the subordinate (restraint models) or by none of the two group members (tug-of-war models). Concession models predict that the dominant should yield just enough reproduction to a subordinate to make it favourable for the latter to stay in the group (Emlen 1982; Vehrencamp 1983; Reeve \& Ratnieks 1993), hence leading to a positive association between relatedness among breeders and skew. Alternatively, restraint models predict that the subordinate should capture the largest share of reproduction that the dominant will tolerate before ejecting the subordinate (Johnstone \& Cant 1999), which is predicted to result in a negative association between relatedness among breeders and skew. Finally, tug-of-war models assume that the conflict over reproductive allocation takes place at the expense of total group productivity (Reeve et al. 1998) and they predict no or a weakly negative correlation between relatedness among breeders and skew but a positive association between relatedness and overall group productivity.
Finally, we investigated whether the pattern of queen specialization was associated with the relatedness between queens and their mate(s). Previous studies showed that a high proportion of $F$. exsecta queens mate with related males (Sundström et al. 2003; C. Haag-Liautard, J. S. Pedersen, O. Ovaskainen \& L. Keller, unpublished data), which may translate in the production of less fit diploid (female) offspring (Charlesworth \& Charlesworth 1987) and a higher probability of producing diploid nonfertile males (Cook \& Crozier 1995; Crozier \& Pamilo 1996). Hence, queens mated with a related male should be more specialized in male production than queens mated with an unrelated male. This might be either due to reduced survival rate of diploid brood produced by queens mated with a related male or because these queens actively choose to produce mostly males to avoid the cost of inbreeding.

\section{METHODS}

\section{Sampling Procedure}

To establish laboratory colonies, we collected $3.0 \pm 0.5$ (mean $\pm \mathrm{SD}$ ) queens and approximately 300 workers from each of 20 field colonies on 29 April 2003 when queens were about to start egg laying. Ants were collected at 'les Chenevières', a cattle pasture at $1220 \mathrm{~m}$ altitude in the Swiss Jura Mountains. In this population, colonies are known to contain many queens (Cherix et al. 1980; Brown \& Keller 2000; Liautard \& Keller 2001). Sex ratio is bimodally distributed (Brown \& Keller 2000) and highly male biased with about $90 \%$ of all colonies producing only male sexuals (along with workers). We created artificial colonies in plastic compartments $(26 \times 41 \mathrm{~cm}$ and $15 \mathrm{~cm})$ by providing peat as nest material and a tile as nesting site. Colonies were kept at $22-24^{\circ} \mathrm{C}$ and regularly fed with honey water and a mixture of agar, egg, honey and water.

Queens started egg laying shortly after colonies were established in the laboratory. After two weeks, we carefully opened 10 randomly chosen colonies out of the 20 colonies to collect eggs for genetic analyses. We collected all queens and pupae and a random sample of workers from the 20 experimental colonies 4 weeks after the egg sampling. To check whether queens were inseminated, we dissected all queens and determined the presence or absence of sperm in their spermathecae.

\section{Laboratory Methods}

All experimental colonies produced males and/or workers (Table 1). We determined the sex and caste of all pupae produced by moistening them in ethanol and observing their size, eye shape and genitalia (Brown \& Keller 2000; Liautard et al. 2003). All queens, the sperm in their spermathecae and eight workers per colony were genotyped at seven microsatellite loci (FL21, Chapuisat 1996 and FE13, FE17, FE19, FE21, FE37, and FE51, Gyllenstrand et al. 2002). The mean number of alleles per locus was high (mean \pm SD: $9.8 \pm 6.8$ ). We also genotyped $17 \pm 10$ (mean \pm SD) male and $22 \pm 12$ worker pupae per colony at the four most variable loci (FL21, FE13, FE17 and 
Table 1. Number of colonies that produced males and/or workers quantified at two different life stages of the brood (egg and pupal stage)

\begin{tabular}{|c|c|c|c|c|c|c|}
\hline Colony ID & $\begin{array}{l}\text { No. of } \\
\text { queens }\end{array}$ & $\begin{array}{c}\text { No. of } \\
\text { inseminated } \\
\text { queens }\end{array}$ & $\begin{array}{l}\text { No. of males } \\
\text { (egg stage)* }\end{array}$ & $\begin{array}{c}\text { No. of workers } \\
\text { (egg stage)* }\end{array}$ & $\begin{array}{l}\text { No. of males } \\
\text { (pupal stage) } \dagger\end{array}$ & $\begin{array}{l}\text { No. of workers } \\
\text { (pupal stage) } \dagger\end{array}$ \\
\hline $\mathrm{C} 1$ & 3 & 3 & 24 & 20 & 81 & 23 \\
\hline C2 & 3 & 1 & 31 & 16 & 138 & 52 \\
\hline C3 & 3 & 1 & - & - & 89 & 1 \\
\hline C4 & 3 & 2 & - & - & 20 & 120 \\
\hline C5 & 3 & 2 & 22 & 6 & 4 & 32 \\
\hline C6 & 3 & 2 & - & - & 17 & 141 \\
\hline C7 & 3 & 2 & - & - & 1 & 143 \\
\hline C8 & 3 & 3 & 32 & 8 & 119 & 2 \\
\hline C9 & 2 & 2 & 1 & 38 & 0 & 130 \\
\hline C10 & 2 & 1 & - & - & 135 & 0 \\
\hline C11 & 4 & 3 & 12 & 34 & 94 & 53 \\
\hline $\mathrm{C} 12$ & 3 & 3 & 6 & 25 & 63 & 68 \\
\hline C13 & 3 & 3 & - & - & 0 & 237 \\
\hline C14 & 3 & 3 & - & - & 92 & 92 \\
\hline C15 & 3 & 3 & - & - & 14 & 193 \\
\hline C16 & 3 & 1 & - & - & 16 & 194 \\
\hline $\mathrm{C} 17$ & 3 & 2 & - & - & 98 & 57 \\
\hline C18 & 3 & 3 & 13 & 24 & 48 & 134 \\
\hline C19 & 2 & 1 & 27 & 13 & 25 & 0 \\
\hline $\mathrm{C} 20$ & 4 & 4 & 0 & 38 & 3 & 177 \\
\hline Mean & 3.0 & 2.3 & 17 & 22 & 53 & 92 \\
\hline SD & 0.5 & 0.9 & 12 & 12 & 49 & 74 \\
\hline
\end{tabular}

*Relative estimates based on the genotyped eggs. Because none of the colonies reared gynes (new queens), we regarded all diploid eggs as worker-destined eggs.

$\dagger$ Absolute estimates based on collected pupae.

FE37). DNA was extracted from the head of adult individuals (workers and queens), the entire pupae and the spermatheca in 250, 500 and $50 \mu \mathrm{l}$ of $5 \%$ Chelex, respectively. Samples were incubated for $10 \mathrm{~min}$ at $90^{\circ} \mathrm{C}$, vortexed, and incubated for another $10 \mathrm{~min}$ at $90^{\circ} \mathrm{C}$. Two multiplex PCR were used that co-amplified the loci FE19, FE21 and FE51 (multiplex-PCR1) and the loci FE17 and FE37 (multiplex-PCR2). The loci FE13 and FL21 were amplified in simplex PCRs. Detailed procedures of amplifying conditions are described elsewhere (Kümmerli et al. 2005). PCR products were mixed and run on an automatic sequencer (ABI Prism 377XL).

For each colony, we calculated the numerical investment into males as the number of male offspring divided by the total number of offspring produced. To determine the numerical investment into males at the egg stage, we genotyped $39 \pm 6$ (mean \pm SD) eggs per colony at the seven microsatellites in 10 colonies. DNA from eggs was extracted using a standard phenol-chloroform-isoamylalcohol (PCI) protocol (Schwander et al. 2006). Eggs that had only one allele at all loci were classified as males, whereas eggs being heterozygote at least at one locus were classified as diploids (workers). This classification was reliable because all queens were mated with males that had at least one diagnostic allele at the loci studied.

\section{Maternity Assignment}

Of the 513 males and 663 workers genotyped (1176 offspring in total), we could unambiguously assign 1152 individuals to one of the colony's queen. Moreover, because queens were unrelated to the workers in the colony $(r=0.03 \pm 0.02)$, the probability that a worker-produced male would be wrongly classified as a queen-produced male is negligible. The genotype of 24 individuals (12 male and 12 worker offspring) were incompatible with the genotype of queens and their mates, hence being individuals that developed from field-collected eggs and/or from workerproduced eggs. All these individuals were excluded from further analyses.

\section{Reproductive Skew}

We estimated reproductive skew among queens for the production of male and worker brood as well as for the total number of brood produced for each colony at the egg and pupal stages by calculating the $B$ index (Nonacs 2000) with the program SKEW CALCULATOR 2003 (http:// www.obee.ucla.edu/Faculty/Nonacs/). The $B$ index is described by

$$
B=\sum_{i=1}^{N}\left(p_{i}-\frac{n_{i}}{N_{t}}\right)^{2}-\left(1-\frac{1}{\bar{N}}\right) / K
$$

where $K$ is the total number of offspring produced, $N$ the number of queens in the nest, $p_{i}$ the proportion of offspring produced by the $i$ th queen, $n_{i}$ the time queen $i$ was present in the colony, and $N_{t}$ the sum of the $n_{i}$ for the colony. $\bar{N}=N_{t} / n_{\max }$ is a weighted mean group size where $n_{\max }$ is the maximum time any queen could be present in the nest. Because all queens were present for the same length of time in our study: $n_{i / N_{\mathrm{t}}}=1 / \mathrm{N}$ and 
$\bar{N}=N$. The $B$ index is 0 for randomly distributed reproduction, positive when skew is greater than random and negative when skew is distributed more evenly than random. We tested whether there were significant differences between the reproductive skew values for the production of males, workers and the total brood and between skew values at the egg and at the pupal stage. To calculate the $B$ index for the production of worker brood, we only used colonies containing at least two mated queens and for the production of male and worker brood, we considered only colonies that produced at least five males or workers (see Table 1 and Fig. 1 for the number of colonies that fit these criteria). Because the number of queens and brood analysed varied across colonies, the expected values of the $B$ index under complete monopoly differed between colonies (range: $0.48-0.73$, mean \pm SD: $0.62 \pm$ 0.06). To make values of reproductive skew comparable between colonies and analyses, we standardized all reproductive skew values by using the ratio of observed to maximal skew.

We estimated relatedness among nestmate queens with the program RELATEDNESS 5.0.8 (Queller \& Goodnight 1989) by using the genotypes of adult workers to calculate the population allele frequencies and tested whether relatedness correlates with the $B$ index. Furthermore, we tested whether relatedness among queens was associated with the mean productivity among queens per colony. To get an estimate of the mean productivity among queens per colony, we multiplied the number of male and worker pupae produced in a colony by their corresponding dry weight (males: $W_{\mathrm{m}}=1.71 \mathrm{mg}$, workers: $W_{\mathrm{w}}=1.13 \mathrm{mg}$; (Brown \& Keller 2000) and divided this value by the number of queens that were present in the colony.

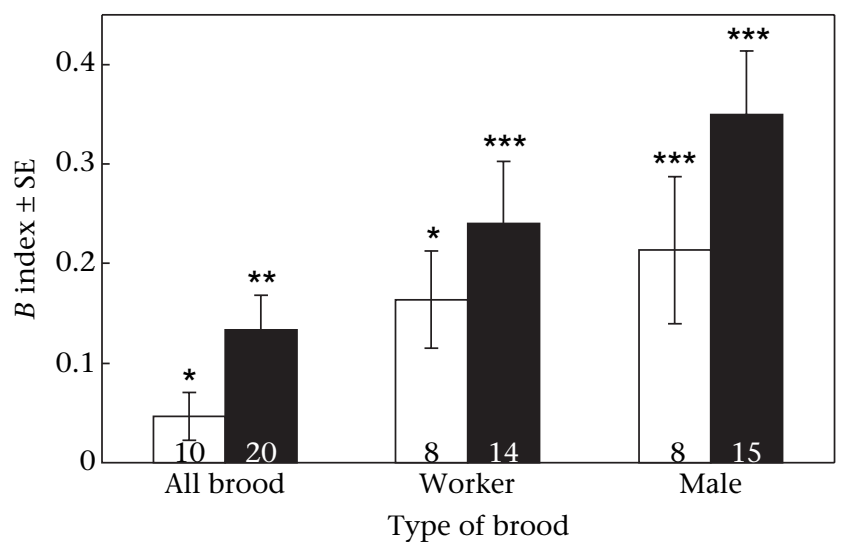

Figure 1. Reproductive skew among nestmate queens measured as the $B$ index (Nonacs 2000). The $B$ index was calculated for the production of all brood as well as for the production of worker and male brood, at the egg $(\square)$ and at the pupal stage ( $\square)$ and was significantly greater than zero for all comparisons $\left({ }^{*} P \leq 0.05,{ }^{* *} P \leq 0.01\right.$, $* * * P \leq 0.001)$. Numbers at the bottom of each column indicate the number of colonies that produced at least five offspring (see Table 1) of the corresponding brood type and were therefore considered for $B$ index calculations.

\section{Reproductive Specialization Among Queens}

We used two different measures to quantify the reproductive specialization of queens. First, we calculated the reproductive specialization of each queen individually as the number of male offspring divided by the total number of offspring she produced. For this measure of reproductive specialization that ranges from 0 (only worker production) to 1 (only male production), we only considered queens that produced at least five offspring, which was the case for 27 out of 29 queens at the egg stage and for 50 out of 59 queens at the pupal stage. Second, we estimated the degree of reproductive specialization across all queens in a colony as the effective proportion of queens $\left(\tau_{\mathrm{c}}\right)$ contributing to either males or workers given by

$$
\tau_{\mathrm{c}}=1-\frac{N_{\mathrm{e}, \mathrm{m}+\mathrm{w}}}{N_{\mathrm{e}, \mathrm{m}}+N_{\mathrm{e}, \mathrm{w}}-N_{\mathrm{e}, \mathrm{m}+\mathrm{w}}}
$$

where $N_{e, m}$ is the effective number of queens contributing to males, $N_{\mathrm{e}, \mathrm{w}}$ the effective number of queens contributing to workers and $N_{\mathrm{e}, \mathrm{m}+\mathrm{w}}$ the effective number of queens contributing to both classes of offspring (Kümmerli \& Keller 2007). This measure includes information on the variance in the reproductive output between queens and on each queen's differential contribution to males and workers, whereby $N_{\mathrm{e}, \mathrm{m}}=\sum n_{\mathrm{m}, \mathrm{Qi}} / n_{\mathrm{m}, \mathrm{Qr}}$ and $N_{\mathrm{e}, \mathrm{w}}=$ $\sum n_{\mathrm{w}, \mathrm{Qi}} / n_{\mathrm{w}, \mathrm{Qr}}$ with $n_{\mathrm{m}, \mathrm{Qi}}$ and $n_{\mathrm{w}, \mathrm{Qi}}$ being the respective number of males and worker produced by the $i$ th queen and $n_{\mathrm{m}, \mathrm{Qr}}$ and $n_{\mathrm{w}, \mathrm{Qr}}$ being the respective number of males and workers produced by the most reproductive queen within a colony. Finally,

$$
N_{\mathrm{e}, \mathrm{m}+\mathrm{w}}=\sum\left[n_{\mathrm{m}, \mathrm{Qi}}+n_{\mathrm{w}, \mathrm{Qi}}-\left|n_{\mathrm{m}, \mathrm{Qi}}-n_{\mathrm{w}, \mathrm{Qi}}\right|\right] / n_{\mathrm{m}+\mathrm{w}, \mathrm{Qr}},
$$

where $n_{\mathrm{m}+\mathrm{w}, \mathrm{Qr}}$ is the sum of the number of males and workers produced by the most reproductive queen within a colony. $\tau_{\mathrm{c}}$ provides a continuous measure of reproductive specialization among queens that ranges from 0 to 1 . At one end of the continuum, a complete lack of specialization would occur if all queens equally contribute to males and workers $\left(\tau_{c}=0\right)$. At the other end of the continuum a complete specialization would occur if each queen exclusively contributes to a single type of individuals $\left(\tau_{\mathrm{c}}=1\right)$.

We calculated the asymmetric relatedness of males to the queens they mated with. In haplodiploid organisms, this relatedness value is equal to $F_{\text {IS }}$ (inbreeding coefficient; Liautard \& Sundström 2005). We first tested whether $F_{\text {IS }}$ was greater than zero and then examined whether $F_{\text {IS }}$ was greater for male-specialized queens (i.e. those who produced mostly males) than for workerspecialized queens (i.e. those who produced mostly workers).

\section{Statistical Analyses}

Data from 10 colonies were available for analysis of brood at the egg stage, whereas data from 20 colonies were analysed for brood at the pupal stage. We used the 10 colonies for which brood was available for both life stages 
for comparisons of reproductive patterns between the egg and pupal stages.

Estimates of relatedness were normalized prior to analysis by applying logarithmic transformations. All other variables (numerical investment into males and workers, the $B$ index and measures of $\tau_{\mathrm{c}}$ ) deviated significantly from normal distributions even after applying arcsine and logarithmic transformations (Shapiro-Wilk's test: $P<0.05$ for all variables). We, therefore, used Spearman's rank correlation coefficient for correlation analyses and permutation analyses (Manly 1997) for one-sample tests and for analysis-of-variance-like designs (ANOVA).

To test whether a sample differed significantly from an expected value (one-sample test), we applied Fisher's onesample randomization test (10000 iterations) using the program RUNDOM PROJECTS 2.0 LITE (http://pjadw. tripod.com). For ANOVA designs, we first applied a classical ANOVA computation on our data set and extracted the obtained $F$ values that we classified as the observed values of our analysis $\left(F_{\text {obs }}\right)$. Whenever appropriate, we introduced the colony as a factor into our model to account for repeated measures taken from the same colony (Sokal $\&$ Rohlf 1995). We then performed 1000 random permutations on our data set and calculated the $F$ values after each permutation using the computer program $\mathrm{R}$ 2.2.0 (http://www.r-project.org). We counted the number of cases in which $F$ values obtained from the permutation were greater or equal to the observed $F$ values $\left(N_{F \geq F_{\text {obs }}}\right)$. Finally, we calculated the probability of getting $F$ values greater or equal to the observed $F$ values by using the formula $P=\left(1+N_{F \geq F_{\text {obs }}}\right) / 1000 . \quad P$ values $\leq 0.05$ were regarded as significant.

\section{RESULTS}

\section{Numerical Investment into Males}

The median proportion of males produced was 0.45 (1st quartile $=0.21$; 3rd quartile $=0.67$ ) at the egg stage and 0.37 (1st quartile $=0.07$; 3rd quartile $=0.74$ ) at the pupal stage. There was no significant difference between these two values (ANOVA permutation test: $N=10, P=0.47$ ). However, there was a significant positive correlation between the colony-level proportion of males at the egg and at pupal stages (Spearman's rank correlation: $\left.r_{\mathrm{S}}=0.66, N=10, P=0.038\right)$.

\section{Reproductive Skew}

The average reproductive skew values for the production of males, workers and all brood at the egg and pupal stages were all significantly greater than 0 (range: $0.05 \leq B$ index $\geq 0.35$, Fisher's one-sample randomization tests: $P \leq 0.05$; Fig. 1). There was a tendency for reproductive skew values to be smaller at the egg than at pupal stage but the differences were not significant (ANOVA permutation tests, all brood: $N=10, P=0.213$; workers: $N=7$, $P=0.094$; males: $N=7, P=0.341$; Fig. 1 ). At the pupal stage, the reproductive skew values were significantly lower for all brood than for males only (ANOVA permutation test: $N=15, P=0.006$ ) and also lower (but not significantly) for all brood than for workers only (ANOVA permutation test: $N=14, P=0.077$ ). The high reproductive skew for male brood was not due to the presence of unmated queens in the colony demonstrated by $\mathrm{B}$ indexes not being significantly different between colonies where all queens were mated $(N=6, B$ index $\pm \mathrm{SE}=$ $0.37 \pm 0.10)$ and colonies where not all queens were mated $(N=9, B$ index $\pm \mathrm{SE}=0.33 \pm 0.09$; ANOVA permutation test: $P=0.79$ ).

Across colonies there was a positive correlation between reproductive skew at the egg and pupal stages. The correlation was marginally significant for worker brood (Spearman's correlation coefficient: $r_{\mathrm{S}}=0.75, N=7, P=$ $0.052)$ and significant for male brood $\left(r_{\mathrm{S}}=0.96, N=7\right.$, $P<0.001)$ and all brood $\left(r_{\mathrm{S}}=0.76, N=10, P=0.011\right)$.

The relatedness (mean $\pm \mathrm{SE}$ ) among nestmate queens was low $\left(r_{\mathrm{q}}=0.06 \pm 0.04\right.$, range: -0.30 to 0.43$)$. There were no significant correlations between the relatedness among queens and the values of reproductive skew for male, worker and all brood production (Spearman's rank correlations: $0.0 \leq r_{\mathrm{S}} \leq 0.29$; all $P>0.42$ ). The mean productivity among queens per colony ranged from 14 to $126 \mathrm{mg}$ (mean $\pm \mathrm{SD}: 79 \pm 26 \mathrm{mg}$ ) and did not significantly correlate with the relatedness among nestmate queens (Spearman's correlation coefficient: $r_{\mathrm{S}}=0.31$, $N=20, P=0.19$ ). Moreover, there were no significant correlations between the mean productivity among queens per colony and the values of reproductive skew for male, worker and all brood production (Spearman's rank correlations: $-0.58 \leq r_{\mathrm{S}} \leq 0.40$; all $P \geq 0.08$ ).

\section{Reproductive Specialization}

A high proportion of the queens that produced at least five offspring were specialized in the production of a single caste. At the egg stage, $74 \%$ of the queens produced more than $90 \%$ workers or males and $67 \%$ produced only individuals of a single offspring class. Similarly, at the pupal stage $92 \%$ of the queens produced more than $90 \%$ workers or males and $84 \%$ produced only individuals of a single offspring class (Fig. 2).

A comparison of the reproductive specialization of the 27 queens for which data were available at the egg and pupal stages revealed a significant positive correlation between the numerical investment into males at the egg and pupal stages (Spearman's correlation coefficient: $r_{\mathrm{S}}=0.85, N=27, P<0.001$; Fig. 3). Moreover, 17 of the 27 queens (63\%) were completely specialized in the production of either males or workers at both life stages.

In line with these results, the effective proportion of queens $\left(\tau_{\mathrm{c}}\right.$, mean $\pm \mathrm{SE}$ ) contributing to a single caste was very high both at the egg $(0.83 \pm 0.05)$ and pupal stages $\left(\tau_{\mathrm{c}}=0.95 \pm 0.03\right.$; Fig. 4$)$. However, the latter value was significantly greater (ANOVA permutation test: $N=10$, $P=0.004)$, indicating that the degree of specialization increased between the egg and pupal stages.

Of the 59 queens analysed, 14 were unmated, 34 were singly mated and 11 were doubly mated. Nine out of the 14 unmated queens produced more than five males and 


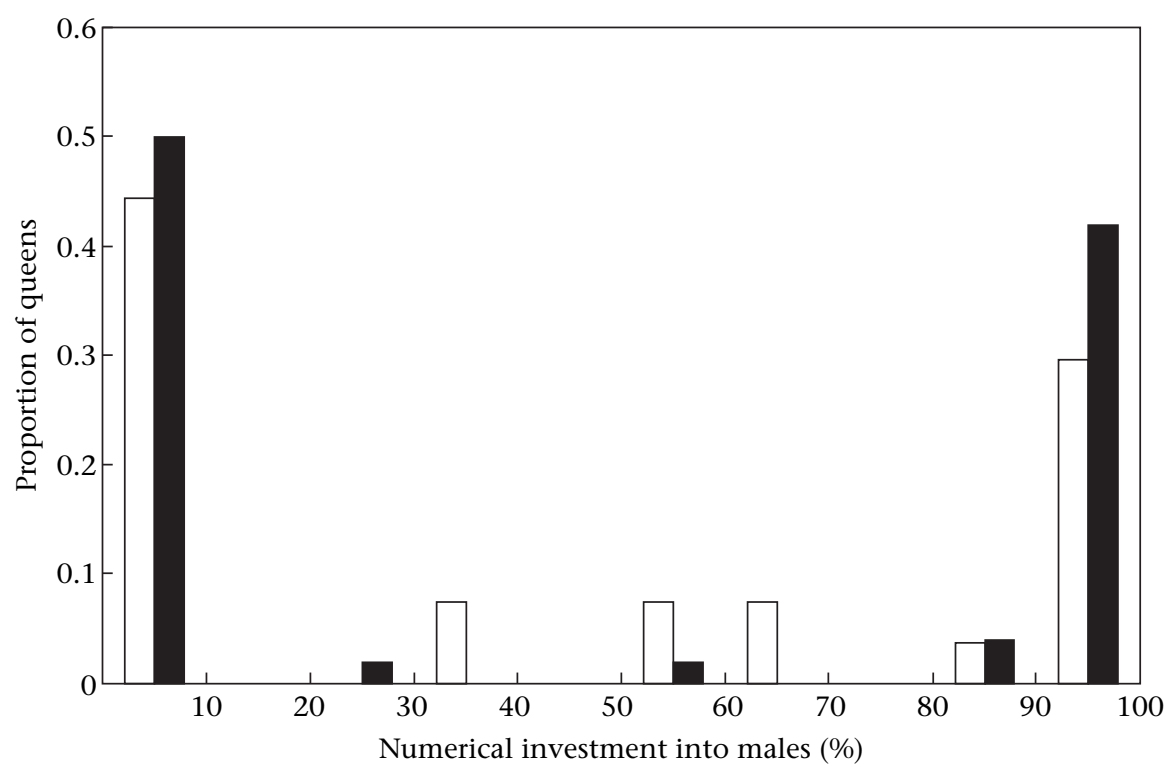

Figure 2. The queens proportional numerical investment into males. Measures are presented for all queens that had more than five offspring ( $\square$ : egg stage: $N=27 ; \mathbf{\square}$ : pupal stage: $N=50$ ).

accounted for $43 \%$ of all queens that invested more than $90 \%$ of their numerical reproduction into males. When unmated queens and the colonies that contained only one inseminated queen were excluded from the analyses, the mean effective proportion of reproductively specialized queens $\left(\tau_{\mathrm{c}}\right)$ was still very high $\left(\tau_{\mathrm{c}}=0.91 \pm 0.06, N=15\right.$; Fig. 4) and did not significantly differ from the estimated $\tau_{\mathrm{c}}$ when all queens were included (ANOVA permutation test: $N=15, P=0.37$ ).

The inbreeding coefficient $\left(F_{\text {IS }}\right)$ between queens and their mates was significantly greater than zero $\left(F_{\mathrm{IS}} \pm \mathrm{SE}=\right.$ $0.15 \pm 0.06$; one-sample $t$ test: $\left.t_{42}=2.68, P=0.005\right)$. However, there was no significant difference in this $F_{\text {IS }}$ value between queens specialized in worker production ( $\left.F_{\text {IS }}=0.16 \pm 0.07, N=26\right)$ and queens specialized in male production $\left(F_{\mathrm{IS}}=0.17 \pm 0.10, N=12 ; t\right.$ test: $t_{36}=$ 0.18, $P=0.86)$. Furthermore, within colonies, the $F_{\text {IS }}$ value for the most productive queen $\left(F_{\mathrm{IS}}=0.12 \pm 0.08\right)$ was not significantly different from that of the queen with the lowest productivity $\left(F_{\mathrm{IS}}=0.06 \pm 0.11\right.$; paired $t$ test: $t_{14}=0.47, P=0.65$ ).

\section{DISCUSSION}

Our brood rearing experiment in F. exsecta laboratory colonies revealed a yet undescribed pattern of reproductive specialization among nestmate queens in ants. Most colonies produced both males and workers but $84 \%$ of the queens contributed exclusively to one type of brood. This pattern of reproduction resulted in very high reproductive skew values ( $B$ index) and an effective proportion of specialized queens within colonies $\left(\tau_{\mathrm{c}}\right)$ being close to 1 .

That ant queens can be specialized in the production of haploid or diploid eggs has previously been reported in Solenopsis invicta (Passera et al. 2001), Pheidole pallidula (de Menten et al. 2005), Formica selysi (Rosset \& Chapuisat
2006) and probably also occurs in Pheidole desertorum (Helms 1999). In these species, however, colonies contained only a single queen or all nestmate queens were specialized in the production of the same type of eggs. Therefore, it was proposed that the pattern of specialization is a strategy by queens to win the conflict that oppose

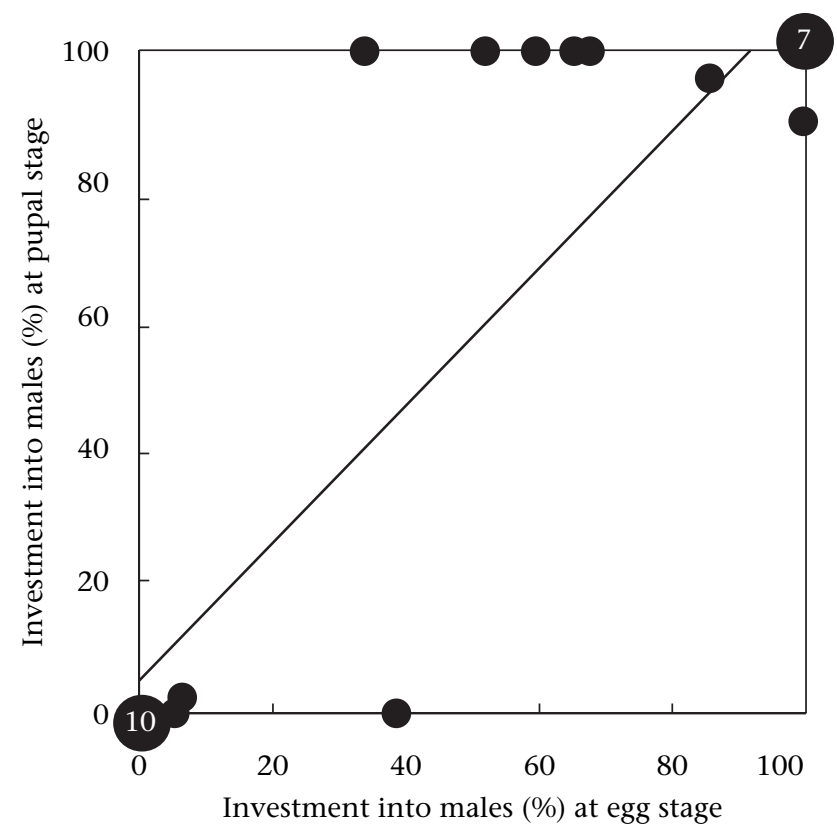

Figure 3. Comparison of reproductive specialization of individual queens $(N=27)$ for brood analysed at the egg and pupal stages measured as the proportion of numerical investment into males. There is a significant positive correlation $(P<0.001)$ between the two values of reproductive specialization indicated by the trendline. Numbers within data points correspond to the number of queens showing the same pattern of reproductive specialization. 


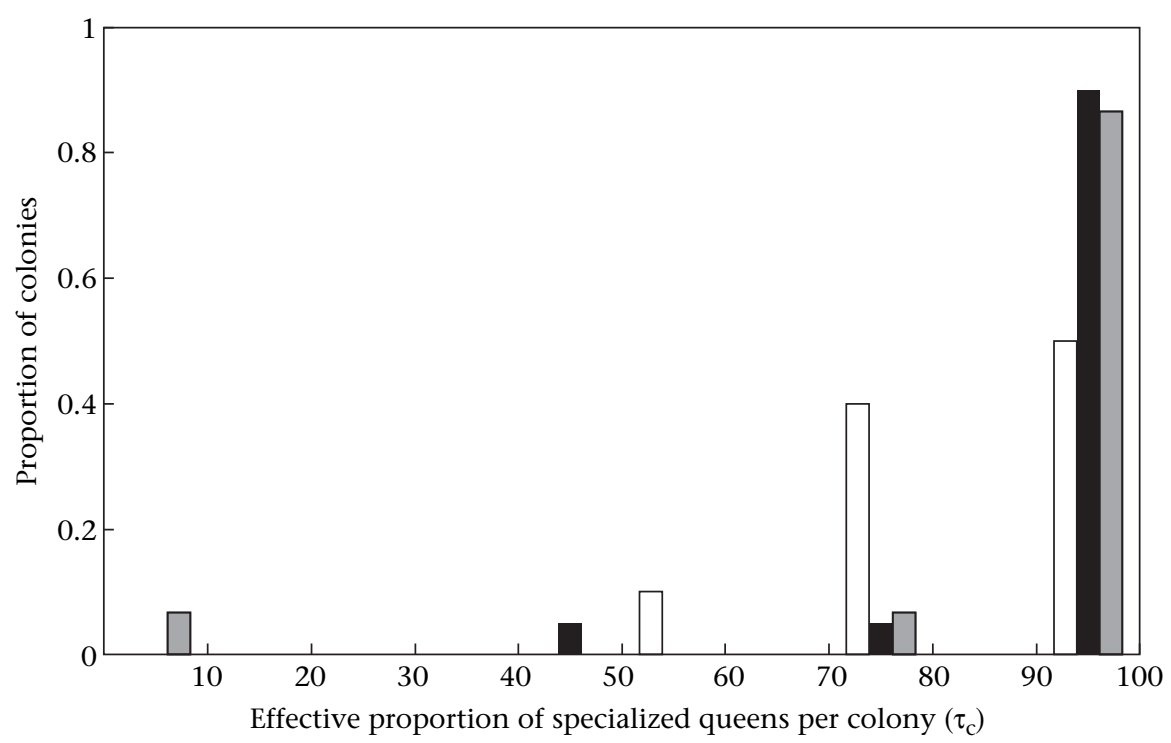

Figure 4. The colonies effective proportion of queens that were specialized in either male or worker production $\left(\tau_{c}\right)$. Measures are presented for colonies including all queens at the egg stage $(N=10, \square)$, at the pupal stage $(N=20, \mathbf{\square})$ and for a reduced data set for the pupal stage that included only the inseminated queens and colonies that hosted at least two inseminated queens $(N=15, \square)$.

them to workers over the optimal allocation of resources into males and females (Trivers \& Hare 1976). By laying only or mostly male eggs in some colonies, queens can force workers to raise males and thus gain fitness benefits when the population sex ratio investment is female biased (Pamilo 1982; Bourke \& Franks 1995; Crozier \& Pamilo 1996). In our study, however, the observed pattern of reproductive specialization among queens is different because differential specialization occurs within and not between colonies.

Our laboratory results are consistent with field data from the same population showing that a large proportion of queens were specialized in male or worker production (Kümmerli \& Keller 2007). However, the estimated proportion of specialized queens was lower in the field $\left(\tau_{c}=0.79\right)$ than in the laboratory $\left(\tau_{\mathrm{c}}=0.95\right)$. Part of this difference may stem from the fact that four laboratory colonies exclusively produced males or workers (with $\tau_{\mathrm{c}}=1$ for these colonies), whereas all field colonies produced males and workers (with $\tau_{\mathrm{c}}<1$ ). Altogether, the high values of $\tau_{\mathrm{c}}$ combined with our finding that $84 \%$ of all queens in the laboratory were completely specialized in the production of either males or workers suggest that the pattern of reproductive specialization detected in the laboratory also occurs in the field.

Two lines of evidence indicate that the observed pattern of reproductive specialization can only partly be explained by the presence of unmated queens that exclusively produced males. First, unmated queens accounted for only $43 \%$ of queens that invested more than $90 \%$ of their numerical reproduction into males, which shows that a considerable proportion $(57 \%)$ of male-specialized queens were inseminated. Second, when removing unmated queens from the analyses, the estimate of the effective proportion of specialized queens in a colony remained very high (91\%).

The comparison of reproductive specialization among nestmate queens at the egg and pupal stages provides information about the proximate mechanisms underlying queen specialization. First, we found that $63 \%$ of all queens contributed exclusively to either males or workers at the egg stage, indicating that the reproductive specialization of most queens was already determined at the egg stage. Second, the effective proportion of specialized queens in a colony significantly increased from the egg to the pupal stage showing that for some queens, reproductive specialization further increased during brood development. This increase in specialization was especially apparent for six queens, which were moderately specialized at the egg stage (33-67\%) but had only male (five queens) or worker (one queen) offspring at the pupal stage (see Fig. 3). There are at least two possible explanations for this. First, workers might selectively remove one type of egg during brood development. Second, some queens produce brood of one sex with extremely low intrinsic viability. Our data do not permit to distinguish between the two explanations, but previous work on F. exsecta showed that workers from polygynous colonies do not discriminate between different types of eggs, but that brood viability significantly differed between queens (Holzer et al. 2006b).

The observed pattern of reproductive specialization might lead to fitness differences between queens if a queen's reproductive specialization remains fixed throughout her life. This is because queens producing only workers would have no direct fitness benefits, while queens specialized in male production would gain direct fitness benefits. Fitness differences between male- and worker-specialized queens might especially occur in the studied polygynous $F$. exsecta population where sex ratio is extremely male biased (Brown \& Keller 2000; Liautard et al. 2003) and most colonies produce only males and workers, but no gynes for several consecutive years (R. Kümmerli \& L. Keller, unpublished data). However, it is yet unknown whether a queen's reproductive specialization is fixed and whether worker-specialized 
queens produce also gynes in gyne-producing colonies. Knowledge about these two points is crucial to address the question of whether reproductive specialization leads to fitness differences between queens.

The lack of a significant association between the degree of reproductive skew and the relatedness among cobreeding queens does not support predictions of either concession or restraint models of reproductive skew. Moreover, the absence of a relationship between colony productivity and queen-queen relatedness are not in line with tug-of-war models, which predict a positive association between these two variables. While reproductive skew models are supported by some data in species of social insects forming small colonies (Reeve \& Keller 2001; Langer et al. 2004, 2006; but see also Liebert \& Starks 2006; Nonacs et al. 2006), our study adds to the growing number of ant forming large colonies where predictions of reproductive skew models are not supported by empirical data (Evans 1996; Bernasconi et al. 1997; Fournier \& Keller 2001; Rüppell et al. 2002; Hannonen \& Sundström 2003; Fournier et al. 2004; Hammond et al. 2006). There are several reasons why reproductive skew models may not be suitable in ants. First, there is considerable variation in queen number in polygynous species and, in colonies containing many queens, it might be difficult for dominant queens to control group membership and the apportionment of reproduction. Second, the presence of workers in highly eusocial insect colonies might affect the dynamics of conflict and reproductive skew among queens (Bourke 2001) because workers have great power over brood rearing (Beekman et al. 2003; Mehdiabadi et al. 2003). Third, there might be only limited options for subordinates to leave the nest to breed solitarily because queens of polygynous ant species have often insufficient energy resources to initiate a nest without workers (Keller \& Passera 1989; Bourke \& Franks 1995; Keller 1995; Chapuisat et al. 1997). Finally, in most ant species the overall colony productivity might be mostly determined by worker number and not by queen number (Keller 1988).

We found that on average queens were significantly related to their mates, confirming that a considerable proportion of matings occurred between colony members (Sundström et al. 2003; C. Haag-Liautard et al., unpublished data). However, the degree of inbreeding (relatedness between the queen and her mates) did not affect the queen's productivity and did not result in diploid male production (unless diploid males died before reaching the pupal stage). Furthermore, queens mated with a related male did not preferentially contribute to male production, suggesting that mated queens do not determine the sex of their progeny so as to circumvent possible negative effects of inbreeding. This finding is consistent with the view that nestmate recognition is poorly developed in highly polygynous ants of the genus Formica (Boomsma et al. 2003; Brown et al. 2003; Holzer et al. 2006a, b), hence possibly preventing queens to distinguish nestmate from non-nestmate males and to regulate the sex of their progeny accordingly.

In conclusion, our study presents a yet undescribed pattern of reproductive specialization among nestmate queens of the ant $F$. exsecta with reproductive skew among queens for egg production being low but with most (84\%) of the queens being completely specialized in the production of either males or workers. This work underlines the importance of investigating reproductive contributions of queens to each type of offspring separately to be able to properly analyse the sharing of reproduction among nestmate queens. However, the ultimate reason for the observed reproductive pattern remains to be investigated because none of the factors studied (the relatedness among queens, colony productivity and the inbreeding status of queens) was correlated with the observed reproductive specialization.

\section{Acknowledgments}

We thank Serge Aron, Ken Helms and two anonymous referees for constructive comments on the manuscript, Cathy Haag-Liautard and David Hughes for useful discussion, Grégoire Castella for feeding the ants and Hélène Jaccard for help in the laboratory. This work was supported by several grants from the Swiss National Science Foundation.

\section{References}

Beekman, M., Komdeur, J. \& Ratnieks, F. L. W. 2003. Reproductive conflicts in social animals: who has power? Trends in Ecology \& Evolution, 18, 277-282.

Bernasconi, G., Krieger, M. J. B. \& Keller, L. 1997. Unequal partitioning of reproduction and investment between cooperating queens in the fire ant, Solenopsis invicta, as revealed by microsatellites. Proceedings of the Royal Society of London, Series B, 264, 1331-1336.

Boomsma, J. J., Nielsen, J., Sundström, L., Oldham, N. J., Tentschert, J., Petersen, H. C. \& Morgan, E. D. 2003. Informational constraints on optimal sex allocation in ants. Proceedings of the $\mathrm{Na}$ tional Academy of Sciences, U.S.A., 100, 8799-8804.

Bourke, A. F. G. 1988. Worker reproduction in the higher eusocial Hymenoptera. Quarterly Review of Biology, 63, 291-311.

Bourke, A. F. G. 2001. Reproductive skew and split sex ratios in social Hymenoptera. Evolution, 55, 2131-2136.

Bourke, A. F. G. \& Franks, N. R. 1995. Social Evolution in Ants. Princeton: Princeton University Press.

Brown, W. \& Keller, L. 2000. Colony sex ratios vary with queen number but not relatedness asymmetry in the ant Formica exsecta. Proceedings of the Royal Society of London, Series B, 267, 1751-1757.

Brown, W. D., Liautard, C. \& Keller, L. 2003. Sex-ratio dependent execution of queens in polygynous colonies of the ant Formica exsecta. Oecologia, 134, 12-17.

Chapuisat, M. 1996. Characterization of microsatellite loci in Formica lugubris B and their variability in other ant species. Molecular Ecology, 5, 599-601.

Chapuisat, M., Goudet, J. \& Keller, L. 1997. Microsatellites reveal high population viscosity and limited dispersal in the ant Formica paralugubris. Evolution, 51, 475-482.

Charlesworth, D. \& Charlesworth, B. 1987. Inbreeding depression and its evolutionary consequences. Annual Review of Ecology and Systematics, 18, 237-268.

Cherix, D., Werner, P. \& Catzeflis, F. 1980. Organisation spatiale d'un système polycalique chez Formica (Coptoformica) exsecta Nyl. (Hymenoptera: Formicidae). Mitteilungen der Schweizerischen Entomologischen Gesellschaft, 53, 163-171.

Cook, J. M. \& Crozier, R. H. 1995. Sex determination and population biology in the Hymenoptera. Trends in Ecology \& Evolution, 10, 281-286.

Crozier, R. \& Pamilo, P. 1996. Evolution of Social Insect Colonies: Sex Allocation and Kin Selection. Oxford: Oxford University Press. 
Emlen, S. T. 1982. The evolution of helping. I. An ecological constraints model. American Naturalist, 119, 29-39.

Evans, J. D. 1996. Competition and relatedness between queens of the facultatively polygynous ant Myrmica tahoensis. Animal Behaviour, 51, 831-840.

Fournier, D. \& Keller, L. 2001. Partitioning of reproduction among queens in the Argentine ant, Lineptihema humile. Animal Behaviour, 62, 1039-1045.

Fournier, D., Aron, S. \& Keller, L. 2004. Significant reproductive skew in the facultatively polygynous ant Pheidole pallidula. Molecular Ecology, 13, 203-210.

Gyllenstrand, N., Gertsch, P. J. \& Pamilo, P. 2002. Polymorphic microsatellite DNA markers in the ant Formica exsecta. Molecular Ecology Notes, 2, 67-69.

Hammond, R. L. \& Keller, L. 2004. Conflict over male parentage in social insects. PLoS Biology, 2, e248.

Hammond, R. L., Bruford, M. W. \& Bourke, A. F. G. 2006. A test of reproductive skew models in a field population of a multiplequeen ant. Behavioral Ecology and Sociobiology, 61, 265-275.

Hannonen, M. \& Sundström, L. 2003. Reproductive sharing among queens in the ant Formica fusca. Behavioral Ecology, 14, 870-875.

Helms, K. R. 1999. Colony sex ratios, conflict between queens and workers, and apparent queen control in the ant Pheidole desertorum. Evolution, 53, 1470-1478.

Holzer, B., Chapuisat, M., Kremer, N., Finet, C. \& Keller, L. 2006a. Unicoloniality, recognition and genetic differentiation in a native Formica ant. Journal of Evolutionary Biology, 19, 2031-2039.

Holzer, B., Kümmerli, R., Keller, L. \& Chapuisat, M. 2006b. Sham nepotism as a result of intrinsic difference in brood viability in ants. Proceedings of the Royal Society of London, Series B, 273, 2049-2052.

Jeon, J. \& Choe, J. C. 2003. Reproductive skew and the origin of sterile castes. American Naturalist, 161, 206-224.

Johnstone, R. A. 2000. Models of reproductive skew: a review and synthesis. Ethology, 106, 5-26.

Johnstone, R. A. \& Cant, M. A. 1999. Reproductive skew and the threat of eviction: a new perspective. Proceedings of the Royal Society of London, Series B, 266, 275-279.

Keller, L. 1988. Evolutionary implications of polygyny in the Argentine ant, Iridomyrmex humilis (Mayr) (Hymenoptera: Formicidae): an experimental study. Animal Behaviour, 36, 159-165.

Keller, L. 1993a. The assessment of reproductive success of queens in ants and other social insects. Oikos, 67, 177-180.

Keller, L. 1993b. Queen Number and Sociality in Insects. Oxford: Oxford University Press.

Keller, L. 1995. Social life: the paradox of multiple-queen colonies. Trends in Ecology \& Evolution, 10, 355-360.

Keller, L. \& Passera, L. 1989. Size and fat content of gynes in relation to the mode of colony founding in ants (Hymenoptera; Formicidae). Oecologia, 80, 236-240.

Keller, L. \& Reeve, H. K. 1994. Partitioning of reproduction in animal societies. Trends in Ecology \& Evolution, 1, 98-102.

Kümmerli, R. \& Keller, L. 2007. Reproductive specialization among nestmate queens in the polygynous ant Formica exsecta. Behavioral Ecology, 18, 375-383.

Kümmerli, R., Helms, K. R. \& Keller, L. 2005. Experimental manipulation of queen number affects colony sex ratio investment in the highly polygynous ant Formica exsecta. Proceedings of the Royal Society of London, Series B, 272, 1789-1794.

Langer, P., Hogendoorn, K. \& Keller, L. 2004. Tug-of-war over reproduction in a social bee. Nature, 428, 844-847.

Langer, P., Hogendoorn, K., Schwarz, M. P. \& Keller, L. 2006. Reproductive skew in the Australian allodapine bee Exoneura robusta. Animal Behaviour, 71, 193-201.
Liautard, C. \& Keller, L. 2001. Restricted effective queen dispersal at a microgeographic scale in polygynous populations of the ant Formica exsecta. Evolution, 55, 2484-2492.

Liautard, C. \& Sundström, L. 2005. Estimation of individual level of inbreeding using relatedness measures in haplodiploids. Insectes Sociaux, 52, 323-326.

Liautard, C., Brown, W. D., Helms, K. R. \& Keller, L. 2003. Temporal and spatial variations of gyne production in the ant Formica exsecta. Oecologia, 136, 558-564.

Liebert, A. E. \& Starks, P. T. 2006. Taming of the skew: transactional models fail to predict reproductive partitioning in the paper wasp Polistes dominulus. Animal Behaviour, 71, 913-923.

Manly, B. F. J. 1997. Randomization, Bootstrap and Monte Carlo Methods in Biology. Chapman and Hall.

Maynard Smith, J. \& Szathmary, E. 1995. The Major Transitions in Evolution. Oxford: Freeman.

Mehdiabadi, N. J., Reeve, H. K. \& Mueller, U. G. 2003. Queens versus workers: sex-ratio conflict in eusocial Hymenoptera. Trends in Ecology \& Evolution, 18, 88-93.

de Menten, L., Fournier, D., Brent, C., Passera, L., Vargo, E. L. \& Aron, S. 2005. Dual mechanism of queen influence over sex ratio in the ant Pheidole pallidula. Behavioral Ecology and Sociobiology, 58, 527-533.

Nonacs, P. 2000. Measuring and using skew in the study of social behavior and evolution. American Naturalist, 156, 577-589.

Nonacs, P., Liebert, A. E. \& Starks, P. T. 2006. Transactional skew and assured fitness return models fail to predict patterns of cooperation in wasps. American Naturalist, 167, 467-480.

Pamilo, P. 1982. Genetic evolution of sex ratios in eusocial Hymenoptera: allele frequency simulations. American Naturalist, 119, 638-656.

Passera, L., Aron, S., Vargo, E. L. \& Keller, L. 2001. Queen control of sex ratio in fire ants. Science, 293, 1308-1310.

Queller, D. C. \& Goodnight, K. F. 1989. Estimating relatedness using genetic markers. Evolution, 43, 258-275.

Reeve, H. K. \& Keller, L. 2001. Tests of reproductive-skew models in social insects. Annual Review of Entomology, 46, 347-385.

Reeve, H. K. \& Ratnieks, F. L. W. 1993. Queen-queen conflict in polygynous societies: mutual tolerance and reproductive skew. In: Queen Number and Sociality in Insects (Ed. by L. Keller), pp. 45-85. Oxford: Oxford University Pess.

Reeve, H. K., Emlen, S. \& Keller, L. 1998. Reproductive sharing in animal societies: reproductive incentives or incomplete control by dominant breeders? Behavioral Ecology, 9, 267-278.

Ross, K. G. 1988. Differential reproduction in multiple-queen colonies of the fire ant Solenopsis invicta (Hymenoptera: Formicidae). Behavioral Ecology and Sociobiology, 23, 341-355.

Rosset, H. \& Chapuisat, M. 2006. Sex allocation conflict in ants: when the queen rules. Current Biology, 16, 328-331.

Rüppell, O., Heinze, J. \& Holldobler, B. 2002. Intracolonial patterns of reproduction in the queen-size dimorphic ant Leptothorax rugatulus. Behavioral Ecology, 13, 239-247.

Schwander, T., Helms Cahan, S. \& Keller, L. 2006. Genetic caste determination in Pogonomyrmex harvester ants imposes costs during colony founding. Journal of Evolutionary Biology, 19, 402-409.

Sokal, R. R. \& Rohlf, F. J. 1995. Biometry. New York: W.H. Freeman and Company.

Sundström, L., Keller, L. \& Chapuisat, M. 2003. Inbreeding and sex-biased gene flow in the ant Formica exsecta. Evolution, 57, 1552-1561.

Szathmary, E. \& Maynard Smith, J. 1995. The major evolutionary transitions. Nature, 374, 227-231.

Trivers, R. L. \& Hare, H. 1976. Haplodiploidy and the evolution of the social insects. Science, 191, 249-263.

Vehrencamp, S. L. 1983. Optimal degree of skew in cooperative societies. American Zoologist, 23, 327-335. 